\title{
Nanotoxicology - An Emerging Discipline Evolving from Nanoparticle Risk Assessment
}

\author{
Nida Tabassum Khan ${ }^{1 *}$ and Samiullah Khan ${ }^{2}$ \\ ${ }^{1}$ Department of Biotechnology, Faculty of Life Sciences and Informatics, Balochistan University \\ of Information Technology, Engineering and Management Sciences, Takatu Campus, Quetta, \\ Balochistan \\ ${ }^{2}$ Department of Biotechnology, Faculty of Life Sciences and Informatics, Balochistan University \\ of Information Technology, Engineering and Management Sciences, Takatu Campus, Quetta, \\ Balochistan \\ *Corresponding Author: Nida Tabassum Khan, Department of Biotechnology, Faculty \\ of Life Sciences and Informatics, Balochistan University of Information Technology, \\ Engineering and Management Sciences, Takatu Campus, Quetta, Balochistan.
}

Received: October 24, 2020

Published: November 30, 2020

(C) All rights are reserved by Nida

Tabassum Khan and Samiullah

Khan.

\begin{abstract}
During this era, applications of nanoparticle has exponentially elevated such as being employed in textiles, pharmaceuticals, cosmetics and medicine. Due to their exclusive properties, nanomaterials are now being considered as an empowering domain that serves as the basis for the development of novel nano-based products in the scientific arena and worth millions in the commercial market. However, this perpetual usage of nanoparticles has intensified numerous environmental and health risks. And therefore, requires attentive evaluation in order to analyze their associated toxicological effects. Thus, investigation of their associated toxicity is a requirement. However, the cumulative diversity of nanomaterials requires to exam a diverse group of nano entities for toxicity prediction subsequently making it a challenging task to gather information regarding their potential exposure and probable risk. Previously employed invitro toxicity testing systems were constrained by principled considerations, time and financial accountabilities Therefore, an alternative unconventional computational approach is needed for the evaluation of nanoparticle associated risk. Such computational approaches for risk assessment are characterized as in silico methods that are cost effective and far less time consuming than customary testing systems and serves to develop a sound infrastructure for nanotoxicology studies.
\end{abstract}

Keywords: Bioactivity; Hazard assessment; In silico; Nanoinformatics; Physiochemical; Surface Functionality

\section{Introduction}

Nanoparticles has enthused numerous stances for future in a variety of scientific and engineering endeavors [1]. Because they exhibit distinctive inherent properties, nanoparticles are employed in diverse array of scientific applications such as being used in pharmaceuticals, electronics, textiles, optics, cosmetics and medicine [2]. During this decade, nanoparticle usage has exponentially increased due to their exclusive properties that they are now being considered as an empowering domain that serves the basis for novel developments in numerous arenas of scientific technology [3]. According to a report published by NSF (National Science Foundation), in 2015 nanotechnology was estimated to be of worth 1 trillion dollars and engineered nanoparticles contributed as a major emergent class of unique nanoscale materials that are being employed into numerous business divisions [4].

However, this interminable usage of nanoparticles has intensified numerous environmental and health risks that requires attentive evaluation in order to analyze their associated toxicological effects for the purpose of designing much safer products [5]. Thus, without encumbering public discernment the ultimate goal is to emphasize on the amalgamation of nanoparticles based products from which commercial market could be benefited and also by carefully assessing their associated environmental risks [6]. 
However, the increasing diversity of nanoparticles means the need to test a wide range of nano entities for toxicity prediction consequently making it challenging to gage the possible exposure and risk of each nanoparticles [7]. Therefore, an alternative computer aided approach for the evaluation of nanoparticle associated hazard is a need in order to develop a sound infrastructure for nanotoxicology studies [8].

Nanotoxicology is the study of toxicity associated with nanoparticles [9]. It is crucial to have information of resultant noxious effects of nanoparticles before employing it in any sort of viable application as the characteristics of nanoparticles are not always well recognized therefore hazard assessment should be done during their fabrication and use $[10,11]$.

The most pertinent merits of nanoparticles which influence their toxicity includes configuration, particle size, distribution, surface functionality and chemical composition [11]. However, all nanoparticles do not generate damaging health effects, it's just when an entity is manipulated at the nanoscale range its size is decreased resulting in alteration in its physiochemical properties, affecting its toxicity potential $[12,13]$.

Subsequently these nano entities exhibits different properties, biological impacts and behaviour comparative to their microscale counterparts $[14,15]$. For instance it was stated that large sized nanoparticles exhibited a more constant behaviour than small sized nanoparticles that displayed differential physiochemical properties [16]. However, nanoparticle that prompt explicit toxicity responses and the mechanism that arbitrates the adversative effects are still principally vague. As a result, countless queries regarding nanoparticle risk assessment still remains a question mark and their toxicity is still a contentious issue [17].

In the EU, the REACH [18], is the existing monitoring body for substance hazard assessment and management which considers nanoparticles as an autonomous complex entities and thus regulate their cataloging and labelling [18]. Moreover, the Nanosafety Cluster, endorsed by the EU commission, assists in monitoring and harmonizing the European activities related to nanoparticles risk assessment [19].
On the other hand, another USA based regulatory body namely EPA (Environmental Protection Agency), has a special directive for nanoparticles known as the TSCA (Toxic Substances Control Act) which considers nanoparticles as unique entities at nanoscale and because of their elevated usage in a variety of commercial products, the regulation implemented by TSCA was protracted to embrace materials fabricated or administered at nanoscale in 2015 [20].

Early stage nanotoxicity identification is a challenging task because of numerous issues such as insufficiency of appropriate datasets, absence of applicable guidelines and incomplete classification of nanoparticles [21]. However, determining nanoparticle related toxicity is required to detect their detrimental consequences on plants, animals, humans and the abiotic surroundings [22].

Previously in vitro models were employed for toxicity testing [23]. But these testing systems are constrained by ethical considerations, extensive time frame, and financial liabilities [24]. Therefore, there was a need for an unconventional computational approach for evaluating the toxicity of useful nanoparticles [25]. Such computational approaches for risk assessment are characterized as in silico methods that are cost effective and far less time consuming than customary testing systems [26]. In addition in silico methods would be a valuable asset for nanotoxicologist as it can anticipate toxicity, based on the results of existing experimental data and could even investigate the extent to which nanoparticles can pose toxic effects on the health of living organisms and its environment [27].

Conversely in silico ways projects suitable platform to implement intellectual analysis strategies for toxicity assessment of nanoparticles [28]. By employing data-driven pathways, in silico methods establish relationships between nanoparticle structure and its physiochemical properties in order to determine their bioactivity profile [29].

As structural similarities of different nanoparticles may produce probable patterns of precise toxicity or biological activity outcomes thus permitting experimental nanoparticles to be categorized by assuming that their structure is responsible for the exhibition of 
their proposed biological activity identified by their structural and compositional topologies such as magnitude, elemental configuration, and functional groups [30]. Such application of informatics in hazard assessment and toxicity determination of nanoparticles is termed as Nanoinformatics [31].

According to Sekhon. [31], the last two decades have witnessed large-scale data explorations in which data-driven experimental and computational approaches with informatics have exploited massive computing networks, cutting-edge information tools, and networking expertise. These approaches were then integrated as a part of an expert system that represents repository of expert knowledge obtained from human experts and allows easy access for data retrieval $[32,33]$. To rationalize the current hazard and risk assessment process in terms of expenditure, it would require the evaluation of specific set of nanoparticles as a whole, without the necessity to exam each member of that set for every regulatory outcome. Moreover, number of animal-based experiments will be greatly reduced with regards to ethical considerations by promoting the use of nanoinformatics in nanotoxicological studies $[34,35]$.

\section{Conclusion}

Thus, nanoinformatics provides an in silico approach for understanding the potential toxicity of different nanomaterials. Besides it improves the coherence of nanotoxicology studies and harmonize regularly used methods. More importantly, nanotoxicology has the potential to address human safety assessment of nano exposure and reduce our reliance on the use of animals for testing.

\section{Bibliography}

1. Nel A., et al. "Nanotechnology environmental, health, and safety issues”. In Nanotechnology Research Directions for Societal Needs in 2020 (2011): 159-220.

2. Sundaram D., et al. "Metal-based nanoenergetic materials: synthesis, properties, and applications". Progress in Energy and Combustion Science 61 (2017): 293-365.

3. Savolainen K. "Nanosafety in Europe 2015-2025: Towards safe and sustainable nanomaterials and nanotechnology innovations". Finnish Institute of Occupational Health (2013).
4. Bainbridge WS. "Nanoscience and Nanotechnology Convergence". In Springer Handbook of Nanotechnology (2017): 1587-1602.

5. Viswanath B and Kim S. "Influence of nanotoxicity on human health and environment: The alternative strategies". In Reviews of Environmental Contamination and Toxicology (2016): 61-104).

6. Tantra R and Shard A. "We need answers". Nature Nanotechnology 8.2 (2013): 71.

7. Martinez-Mayorga K., et al. "The impact of chemoinformatics on drug discovery in the pharmaceutical industry". Expert Opinion on Drug Discovery 15.3 (2020): 293-306.

8. Wani MY., et al. "Nanotoxicity: dimensional and morphological concerns". Advances in Physical Chemistry (2011).

9. Dekkers S., et al. "Towards a nanospecific approach for risk assessment". Regulatory Toxicology and Pharmacology 80 (2016): 46-59.

10. Mumtaz MM., et al. "Introduction to mixtures toxicology and risk assessment". Principles and practice of mixtures toxicology. Wiley-VCH, Weinheim 25 (2010): 1-25.

11. Gajewicz A., et al. "Towards understanding mechanisms governing cytotoxicity of metal oxides nanoparticles: Hints from nano-QSAR studies". Nanotoxicology 9.3 (2015): 313-25.

12. Sohaebuddin SK., et al. "Nanomaterial cytotoxicity is composition, size, and cell type dependent". Particle and Fibre Toxicology 7.1 (2010): 22.

13. Grammatikopoulos P., et al. "Nanoparticle design by gas-phase synthesis". Advances in Physics: X 1.1 (2016): 81-100.

14. Kim JY., et al. "Quantitative structure-activity and quantitative structure-property relationship approaches as alternative skin sensitization risk assessment methods". Journal of Toxicology and Environmental Health, Part A 82.7 (2019): 447-72.

15. Johnston LJ., et al. "Key challenges for evaluation of the safety of engineered nanomaterials". NanoImpact (2020): 100219.

16. Adams LK., et al. "Comparative eco-toxicity of nanoscale TiO2, SiO2, and ZnO water suspensions". Water Research 40.19 (2006): 3527-3532. 
17. Gajewicz A., et al. "Advancing risk assessment of engineered nanomaterials: Application of computational approaches". Advanced Drug Delivery Reviews 64.15 (2012): 1663-1693.

18. Regulation EC. No 1907/2006 of the European Parliament and of the Council of 18 December 2006 concerning the Registration. Evaluation, Authorisation and Restriction of Chemicals (REACH), establishing a European Chemicals Agency, amending Directive 45 (1999): 1-849.

19. Riediker M. "Coordination and collaboration in European research towards healthy and safe nanomaterials". In Journal of Physics: Conference Series 304.1 (2011): 012001.

20. Rodríguez-Ibarra C., et al. "International landscape of limits and recommendations for occupational exposure to engineered nanomaterials". Toxicology Letters (2020).

21. Yosefu NO. "Computational modelling for prediction of nanomaterial toxicity (Doctoral dissertation, Doctoral dissertation, Makerere University)".

22. Boverhof DR and David RM. "Nanomaterial characterization: considerations and needs for hazard assessment and safety evaluation". Analytical and Bioanalytical Chemistry 396.3 (2010): 953-961.

23. Hubal EA. "Biologically relevant exposure science for 21st century toxicity testing". Toxicological Sciences 111.2 (2009): 226-32.

24. Krewski D., et al. "Toxicity testing in the 21st century: implications for human health risk assessment. Risk Analysis". An International Journal 29.4 (2004): 474-479.

25. Abbott A. "Toxicity testing gets a makeover". Nature 461.7261 (2009): 158.

26. Richard A., et al. "New horizons in predictive toxicology: current status and application". Royal Society of Chemistry (2011).

27. Molander L., et al. "Science in Risk Assessment and Policy (SciRAP): an online resource for evaluating and reporting in vivo (eco) toxicity studies". Human and Ecological Risk Assessment: An International Journal 21.3 (2015): 753-762.
28. Belkadi F., et al. "Knowledge-based platform for traceability and simulation monitoring applied to design of experiments process: an open source architecture". Journal of Engineering Design 30 (2019): 311-335.

29. Merlot C. "Computational toxicology-a tool for early safety evaluation”. Drug Discovery Today 15 (2010): 16-22.

30. Bender A and Brown N. "Cheminformatics in Drug Discovery". ChemMedChem 13.6 (2018): 467-469.

31. Sekhon BS. "Nanoinformatics: An Overview". Research and Reviews: A Journal of Pharmaceutical Science 5.1 (2019): 21-5.

32. Yang H., et al. "Computational approaches to identify structural alerts and their applications in environmental toxicology and drug discovery". Chemical Research in Toxicology (2020).

33. Worth A., et al. "Alternative methods for regulatory toxicologya state-of-the-art review". European Commission's Science and Knowledge Service 26797 (2014): 1-470.

34. Worth AP. "The role of QSAR methodology in the regulatory assessment of chemicals". In Recent Advances in QSAR Studies (2010): 367-382.

35. Delrue N., et al. "The adverse outcome pathway concept: A basis for developing regulatory decision-making tools". Alternatives to Laboratory Animals 44.5 (2016): 417-429.

\section{Assets from publication with us}

- Prompt Acknowledgement after receiving the article

- Thorough Double blinded peer review

- Rapid Publication

- Issue of Publication Certificate

- High visibility of your Published work

Website: https://www.actascientific.com/

Submit Article: https://www.actascientific.com/submission.php

Email us: editor@actascientific.com

Contact us: +919182824667 